\title{
On the transition pattern of the oblique detonation structure
}

\author{
Hong Hui Teng $\dagger$ and Zong Lin Jiang \\ State Key Lab of High Temperature Gas Dynamics, Institute of Mechanics, Chinese Academy of \\ Sciences, Beijing, 100190, China
}

(Received 8 February 2012; revised 31 July 2012; accepted 24 September 2012; first published online 26 October 2012)

Oblique detonation waves are simulated to study the evolution of their morphology as gasdynamic and chemical parameters are varied. Although two kinds of transition pattern have previously been observed, specifically an abrupt transition and a smooth one, the determining factors for the transition pattern are still unclear. Numerical results show that the transition pattern is influenced by the inflow Mach number, chemical activation energy and heat release. Despite the fact that these parameters were known to influence the detonation instability, the transition pattern variation cannot be predicted according to the instability criterion. In this study, the difference in the oblique shock and detonation angles is proposed as the criterion to determine the transition pattern with the aid of shock-polar analysis. It is found that the smooth transition will appear when the angle difference is small, while the abrupt transition will occur when the difference is large. The shift from the smooth transition to the abrupt transition occurs when the angle difference is about $15^{\circ}-18^{\circ}$. The previously proposed criterion using the characteristic time ratio is also examined and compared with the present angle difference criterion, and the latter is proved to provide better results.

Key words: detonations, reacting flows

\section{Introduction}

Detonation waves are supersonic combustion waves induced by shock ignition and sustained by the subsequent post-shock heat release. In recent years, the potential application of detonation waves in hypersonic propulsion systems has been widely explored, see Kailasanath (2003) and Roy et al. (2004). One kind of detonation propulsion utilizes the oblique detonation wave, which is the basis of oblique detonation wave engines (ODWE) and Ram Accelerators (Nettleton 2000). To date there remain several challenges in the development of ODWE, among which is to form the necessary stationary oblique structure in the supersonic flow. Hence, it is still of great interest to carry out research on the basic structure of oblique detonation waves with a view to further its application.

In the early research, the oblique detonation structure was usually simplified into an oblique shock wave with an instantaneous post-shock heat release. Li, Kailasanath \& Oran (1994) later simulated the oblique detonation and described the structure as composed of a non-reactive oblique shock, an induction region, a set of deflagration waves, and the oblique detonation surface. The transition of oblique shock wave to 
oblique detonation wave (OSW-ODW) is achieved abruptly by a multi-wave point. Furthermore, this structure is found to be stable and resilient to the inflow disturbances according to the numerical results.

Viguier et al. (1997) studied the oblique detonations in hydrogen-air mixtures experimentally and the results were in good agreement with the numerical results by Li et al. (1994). More recently, Fusina, Sislian \& Parent (2005) studied the behaviour of standing oblique detonation waves near the Chapman-Jouguet (CJ) point of this structure. Daimon \& Matsuo (2004) also discussed its analogy with the onedimensional piston-supported unsteady detonation. However, the morphology of the oblique detonation wave is known to be more complex and some other structures have been observed. For instance, Papalexandris (2000) has shown numerically that triple points may appear on the detonation surface in gas mixtures with high activation energy. These triple points have been observed before in experiments, but nonetheless attracted little attention. Choi et al. (2007) also performed a high-resolution study and the results reveal some small-scale structure, which is similar to the unstable cell of a normal gaseous detonation. Furthermore, Choi, Shin \& Jeung (2009) analysed the coupling relation between the shock and the combustion, and the chemical time and flow time are used to explain the structure evolution. Experimentally, Maeda et al. (2011) recently studied the oblique detonation induced by a hypersonic spherical projectile and the 'straw hat' type of oblique detonation waves is observed. This structure is similar to the structure proposed by Li et al. (1994), although the oblique shock before the transition becomes a curved one.

The structure proposed by Li et al. (1994) has often been used in many subsequent investigations as the basis of the oblique detonation structure. However, it is possible to form another primary structure, where the OSW-ODW transition can be achieved smoothly. Broda (1993) seemingly observed the smooth transition in experiments, but the results are obscure. The smooth transition was later confirmed by Vlasenko \& Sabelnikov (1995) by numerical simulation, but it still lacks deep investigation. Figueira da Silva \& Deshaies (2000) studied the transition distance through numerical simulations and a shock-polar analysis, and found that the smooth transition appears when the induction time becomes trivial compared to the total chemical reaction time. Papalexandris (2000) pointed out that the leading shock curves smoothly until it reaches a final angle for moderate wedge angles. However, the OSW-ODW transition has not been studied in detail and its decisive factors are still unclear. Since the smooth transition will induce a completely different flow field, a study on the transition pattern is of significance and helpful in ODWE design. In this paper, a numerical study is carried out to simulate the OSW-ODW transition phenomenon with variation of different gasdynamic and chemical parameters. Based on the numerical results, a criterion for the transition pattern is proposed and discussed.

\section{Mathematical model and numerical method}

A schematic of the oblique detonation wave induced by a wedge in a combustible gas mixture is shown in figure 1 . The supersonic flow of the combustible gas mixture which reflects from the two-dimensional wedge generates an oblique shock wave first. The shock wave may then induce an exothermic chemical reaction resulting in a complex oblique detonation structure. The computation is carried out in the dashed zone, with the rotated coordinate system along the wedge surface. Previous results ( $\mathrm{Li}$ et al. 1994; Figueira da Silva \& Deshaies 2000) demonstrate that the viscosity and boundary layer have little effect on this structure other than changing the boundary layer thickness slightly; and most of the research carried out before has been based 


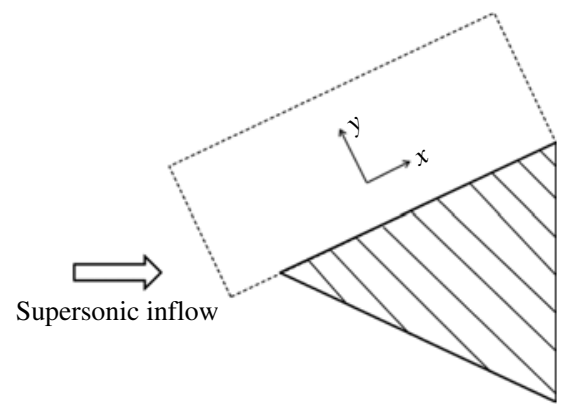

FIGURE 1. A schematic of the oblique detonation wave induced by a wedge in a combustible gas mixture. The computation is carried out in the dashed zone.

on the inviscid assumption. Similarly in the present study, the non-dimensional Euler equations are used as the governing equations, given by

$$
\begin{gathered}
\frac{\partial \boldsymbol{U}}{\partial t}+\frac{\partial \boldsymbol{F}}{\partial x}+\frac{\partial \boldsymbol{G}}{\partial y}+\boldsymbol{S}=0, \\
\boldsymbol{U}=\left[\begin{array}{c}
\rho \\
\rho u \\
\rho v \\
\rho e \\
\rho f \\
\rho g
\end{array}\right], \quad \boldsymbol{F}=\left[\begin{array}{c}
\rho u \\
\rho u^{2}+p \\
\rho u v \\
\rho u(e+p) \\
\rho u f \\
\rho u g
\end{array}\right], \quad \boldsymbol{G}=\left[\begin{array}{c}
\rho v \\
\rho u v \\
\rho v^{2}+p \\
\rho v(e+p) \\
\rho v f \\
\rho v g
\end{array}\right], \quad \boldsymbol{S}=\left[\begin{array}{c}
0 \\
0 \\
0 \\
0 \\
\rho w_{1} \\
\rho w_{2}
\end{array}\right],
\end{gathered}
$$

where the $\rho, u, v, e, p$ are the density, $x$-direction velocity, $y$-direction velocity, total energy and pressure, respectively; variables $f$ and $g$ are the mass fraction of the fuel and intermediate radical. Assuming a perfect gas with constant specific heat ratio $\gamma$, the specific total energy and the equation of state are given by

$$
\begin{gathered}
e=\frac{p}{(\gamma-1) \rho}+\frac{1}{2}\left(u^{2}+v^{2}\right)-q, \\
p=\rho T, \\
q=(1-f-g) Q,
\end{gathered}
$$

where $T$ is temperature and $Q$ is the total heat release. All the variables above are non-dimensionalized through the initial stationary gas parameters, which can be written as

$\rho=\frac{\bar{\rho}}{\bar{\rho}_{0}}, \quad p=\frac{\bar{p}}{\gamma \bar{p}_{0}}, \quad T=\frac{\bar{T}}{\gamma \bar{T}_{0}}, \quad u=\frac{\bar{u}}{\gamma \bar{c}_{0}}, \quad e=\frac{\bar{e}}{\gamma \bar{c}_{0}^{2}}, \quad Q=\frac{\bar{Q}}{\gamma \bar{c}_{0}^{2}}, \quad \bar{c}_{0}^{2}=\frac{\gamma \bar{p}_{0}}{\bar{\rho}_{0}}$,

where $c_{0}$ is the sonic velocity before the shock. The length and time reference scales are defined in the chemical reaction model proposed by Short \& Quirk (1997). The chemical kinetic model is the chain-branching reaction model, which is composed of three parallel steps:

(i) chain initiation: $F \rightarrow Y$;

(ii) chain branching: $F+Y \rightarrow 2 Y$;

(iii) chain termination: $Y \rightarrow P$, 
where $F, Y$ and $P$ correspond to the amount of reactant, radical and product, respectively. The reaction-rate constants are given by

$$
k_{I}=\exp \left(E_{I}\left(\frac{1}{T_{I}}-\frac{1}{T}\right)\right), \quad k_{B}=\exp \left(E_{B}\left(\frac{1}{T_{B}}-\frac{1}{T}\right)\right), \quad k_{T}=1 .
$$

The reference length scale $\bar{x}_{0}$ is chosen so that the chain-termination-rate constant is unity. Therefore the reference time scale is set to be the reference length scale divided by the reference velocity scale. This reaction model can be coupled with the governing equations through

$$
w_{1}=-\rho\left(f k_{I}+f g k_{B}\right), \quad w_{2}=\rho\left(f k_{I}+f g k_{B}-g\right) .
$$

The governing equations are solved on the Cartesian orthogonal uniform grid with the MUSCL-Hancock scheme (Toro 1999). This scheme achieves the second-order accurate in space and time by constructing the Riemann problem on the intercell boundary through the equations:

$$
\left.\begin{array}{l}
\overline{\boldsymbol{U}}_{i}^{L}=\boldsymbol{U}_{i}^{L}+\frac{\Delta t}{2 \Delta x}\left[\boldsymbol{F}\left(\boldsymbol{U}_{i}^{L}\right)-\boldsymbol{F}\left(\boldsymbol{U}_{i}^{R}\right)\right], \\
\overline{\boldsymbol{U}}_{i}^{R}=\boldsymbol{U}_{i}^{R}+\frac{\Delta t}{2 \Delta x}\left[\boldsymbol{F}\left(\boldsymbol{U}_{i}^{L}\right)-\boldsymbol{F}\left(\boldsymbol{U}_{i}^{R}\right)\right] .
\end{array}\right\}
$$

The solution is computed by the HLLC approximate Riemann solver. The default parameters in the chemical reaction model follow previous simulations (Short \& Quirk 1997; $\mathrm{Ng} \&$ Lee 2003), and are $Q=8.33, E_{I}=37.5, E_{B}=10.0, T_{I}=3.0 T_{S} . T_{S}$ is the post-shock temperature, and the ratio of $T_{B}$ and $T_{S}$ is the main bifurcation parameter of the detonation instability. Furthermore the heat release $Q$ and activation energy $E_{B}$ are also used as bifurcation parameters.

\section{Detonation instability characteristics}

To investigate the relation between the transition pattern and the detonation instability, one-dimensional detonation waves are simulated first. Initial conditions are given by the theoretical CJ detonation waves with different cross-over temperature ratios $T_{B} / T_{S}$ placed on the left boundary. After the initial transient the instability develops due to the numerical errors, and subsequently the detonation wave displays various oscillation behaviours. The envelopes of the pressure peaks are shown in figure 2 . When the ratio $T_{B} / T_{S}$ is 0.88 , the detonation will propagate with a decaying oscillation, as shown in figure $2(a)$. The detonation becomes unstable on gradually raising the cross-over temperature ratio. A single-mode oscillation will appear with the ratio 0.90 as shown in figure $2(b)$, and a double-mode oscillation will appear with the ratio 0.92 as shown in figure $2(c)$. When the ratio becomes 0.93 , the oscillation appears irregular and approaches chaos as shown in figure $2(d)$. If the ratio rises further the detonation will quench. For the convenience of discussion, the stable, single-mode oscillation and double-mode oscillation detonations are called Mode 1, Mode 2, Mode 3, respectively. These results are essentially the same as previous results ( $\mathrm{Ng} \&$ Lee 2003) even on doubling the grid resolution. Although detonation instability is very sensitive to the grid resolution (Sharpe 2001), it can be concluded that the grid resolution used in this study is accurate enough to simulate the oblique detonation structures. 

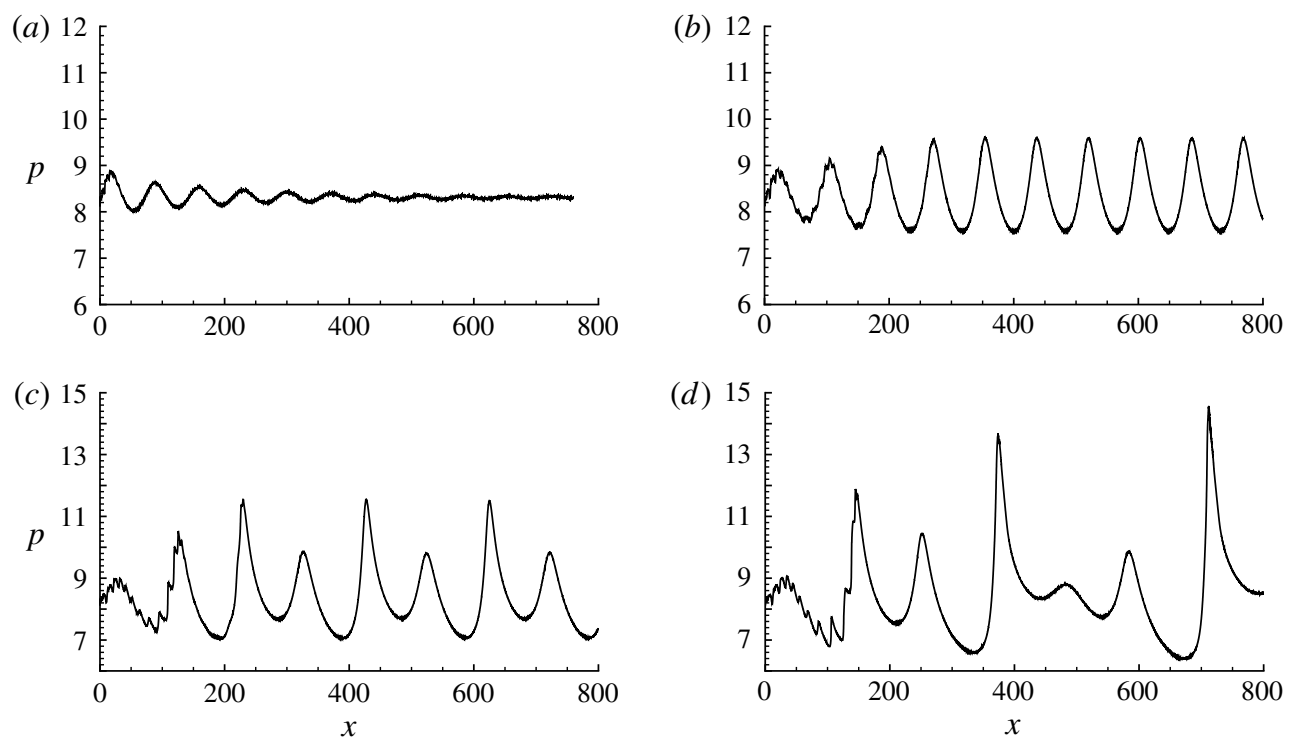

FIGURE 2. Post-shock pressure evolution of one-dimensional pulsed detonation with the cross-over temperature ratio: $(a) 0.88 ;(b) 0.90 ;(c) 0.92 ;(d) 0.93$.

\section{Parametric study on the transition patterns}

Oblique detonation structures are simulated with various gasdynamic and chemical parameters. The inflow conditions are fixed at the free-stream values in both the left and upper boundaries of the domain. Slip boundary conditions are used on the wedge surface, which starts from $x=0.5$ on the lower boundary. The outflow conditions are extrapolated from the interior, and they are implemented on the right and lower boundaries before $x=0.5$ on the wedge. The wedge angle is fixed to be $30^{\circ}$, and the CFL number is fixed at 0.15 . Because the governing equations are non-dimensionalized through the initial gas parameters, the pressure and density are prescribed. The effects of different kinds of gas mixtures are taken into consideration by varying the chemical reaction parameters. In the simulation, the velocity is calculated according to the inflow Mach number $M_{\text {in }}$ first, and then projected onto the rotated coordinate system. The initial flow field is uniform and the non-stationary process is not studied. All the flow fields are calculated until they reach their final stationary states. To compare the results with different heat release, the CJ detonation Mach number $M_{C J}$ is introduced to normalize $M_{i n}$. In the case of $M_{i n} / M_{C J}=1.40$, the structure is shown in figure 3, and it is the abrupt one with an interior shock. In the case of $M_{i n} / M_{C J}=1.50$, the structure is shown in figure 4 , and is obtained from a smooth transition. The smooth transition is achieved by an arc shock, and there is no multi-wave point on the detonation surface. To ensure the resolution is sufficient, another simulation with finer grids is shown in figure 5. It is found that the results are very close and the difference is hardly noticeable. Therefore, one can conclude that the grid is fine enough to produce reliable results and the study on the transition pattern can be carried out.

More cases are simulated between the $M_{\text {in }} / M_{C J}$ ratios 1.40 and 1.50 . Numerical results demonstrate that the change from the abrupt transition to the smooth transition occurs when $M_{i n} / M_{C J}$ changes from 1.44 to 1.45 , as shown in figure 6 . Although the 

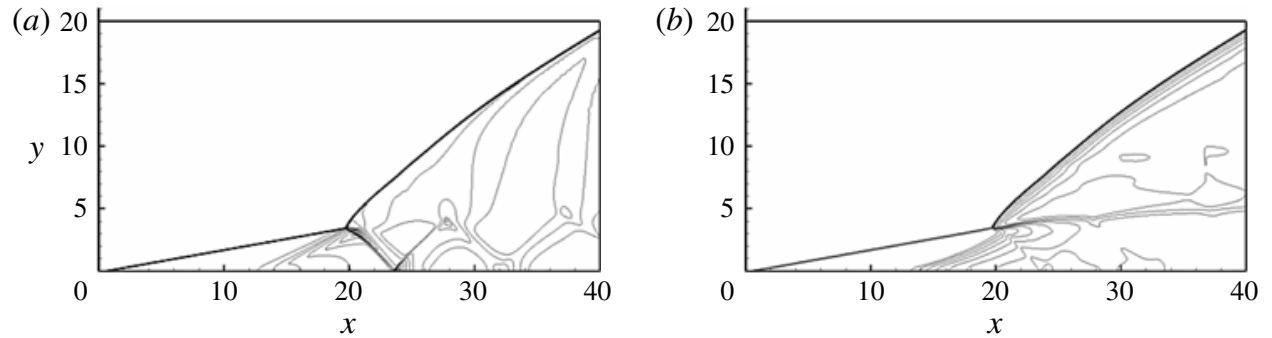

FIGURE 3. (a) Pressure and (b) temperature of the oblique detonation structure with inflow Mach ratio 1.4 and cross-over temperature ratio 0.88 .
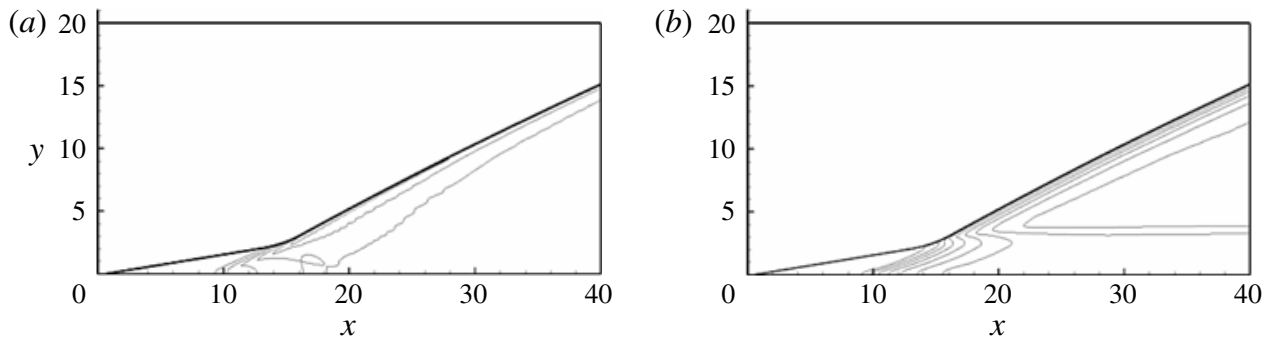

FIgURE 4. (a) Pressure and (b) temperature of the oblique detonation structure with inflow Mach ratio 1.5 and cross-over temperature ratio 0.88 .

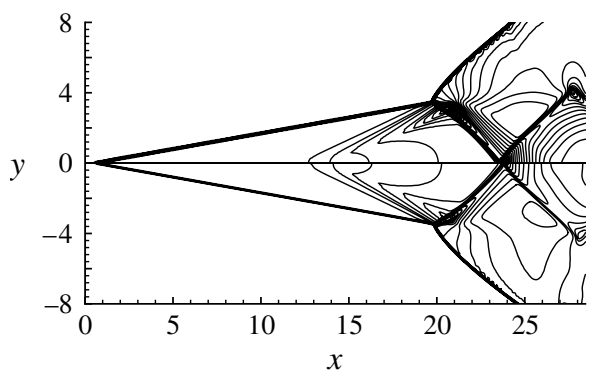

FIGURE 5. Resolution test for the case of inflow Mach ratio 1.4 and cross-over temperature ratio 0.88: grid 0.01 (upper) and grid 0.005 (lower).
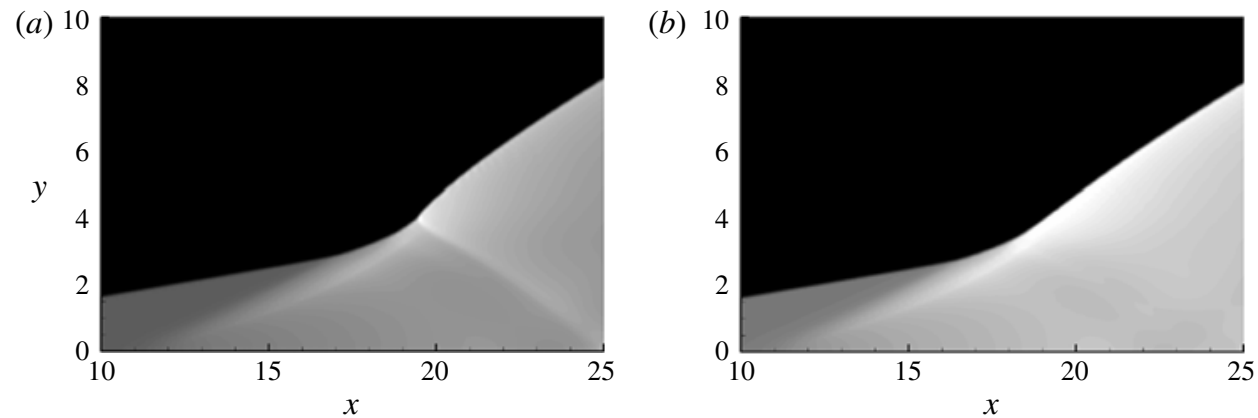

FIgURE 6. Pressure of the oblique detonation structure with inflow Mach ratio $(a) 1.44$ and (b) 1.45 . 


$\begin{array}{lrrrrr}\begin{array}{l}\text { Case } \\ \text { no. }\end{array} & T_{B} / T_{S} & E_{B} & Q & \text { Stability } & M_{c r} \\ 1 & & & & & \\ 2 & 0.88 & 10.0 & 8.33 & \text { Mode 1 } & 1.44 \\ 3 & 0.90 & 10.0 & 8.33 & \text { Mode 2 } & 1.46 \\ 4 & 0.92 & 10.0 & 8.33 & \text { Mode 3 } & 1.48 \\ 5 & 0.88 & 8.0 & 8.33 & \text { Mode 1 } & 1.45 \\ 6 & 0.90 & 8.0 & 8.33 & \text { Mode 1 } & 1.47 \\ 7 & 0.92 & 8.0 & 8.33 & \text { Mode 2 } & 1.49 \\ 8 & 0.88 & 12.0 & 8.33 & \text { Mode 1 } & 1.42 \\ 9 & 0.88 & 14.0 & 8.33 & \text { Mode 2 } & 1.41 \\ 10 & 0.88 & 16.0 & 8.33 & \text { Mode 3 } & 1.40 \\ 11 & 0.90 & 10.0 & 10.00 & \text { Mode 2 } & 1.47 \\ 12 & 0.94 & 10.0 & 15.00 & \text { Mode 2 } & 1.49 \\ & 0.98 & 10.0 & 20.00 & \text { Mode 2 } & 1.51\end{array}$

TABLE $1 . M_{c r}$ and the stability of the detonation wave with different parameters.

difference of this Mach number ratio is only 0.01 , the different transition patterns can be observed clearly. For convenience, the $M_{i n} / M_{C J}$ ratio 1.44 is defined as the critical Mach ratio $M_{c r}$, which corresponds to the upper limit of the abrupt transition.

The $M_{c r}$ variation is studied with different chemical reaction parameters. There are 12 different cases studied as shown in table 1 , with various $T_{B} / T_{S}, E_{B}$ and $Q$. Generally $M_{c r}$ increases either when $T_{B} / T_{S}$ increases, $E_{B}$ decreases, or $Q$ increases. The first case corresponds the results shown in figure 4 to figure 6. From case 1 to case $3, M_{c r}$ increases when the detonation becomes unstable. For the different $E_{B}$ of 8.0 shown in case 4 to case 6 , the detonation instability changes but the $M_{c r}$ trend remains the same. In case 7 to case 9 when $E_{B}$ changes from 12.0 to 16.0 , the detonation becomes unstable but $M_{c r}$ decreases, which is different from the results of case 1 to case 3 . This demonstrates that the transition has a different physical essence from the detonation instability, even though they are both dependent on the same chemical reaction parameters. To investigate the influence of the heat release, case 10 to case 12 are simulated. $E_{B}$ is set to be 10.0 , and one-dimensional detonation is first carried out to make sure the detonation is a single-mode oscillation ('Mode 2') to compare with case 2 . Due to the heat release changing, $M_{C J}$ increases from 3.04 to 4.42. Although the change of $M_{c r}$ seems small, the real inflow $M_{i n}=M_{c r} M_{C J}$ changes considerably in these cases.

\section{Discussion on the transition patterns}

Numerical simulations are performed for different $T_{B}, E_{B}$ and $Q$ to ascertain the criterion for determining the two transition patterns. According to table 1 , the transition patterns cannot be associated with the detonation instability directly despite the fact that they both depend on the same parameters. Since the detonation instability is mainly related to the local structures around the transition region, it is perhaps more natural to discuss the transition pattern basing on the global structure. Due to the heat release, the detonation surface always has a larger oblique angle than the shock surface. As a result, the OSW-ODW transition can be viewed as the oblique angle increasing process. The difference in the shock and detonation oblique angles is therefore the key parameter of the transition from the viewpoint of the global structure. Following the fluid conservation laws, the oblique shock wave angle $\beta_{1}$ is determined 

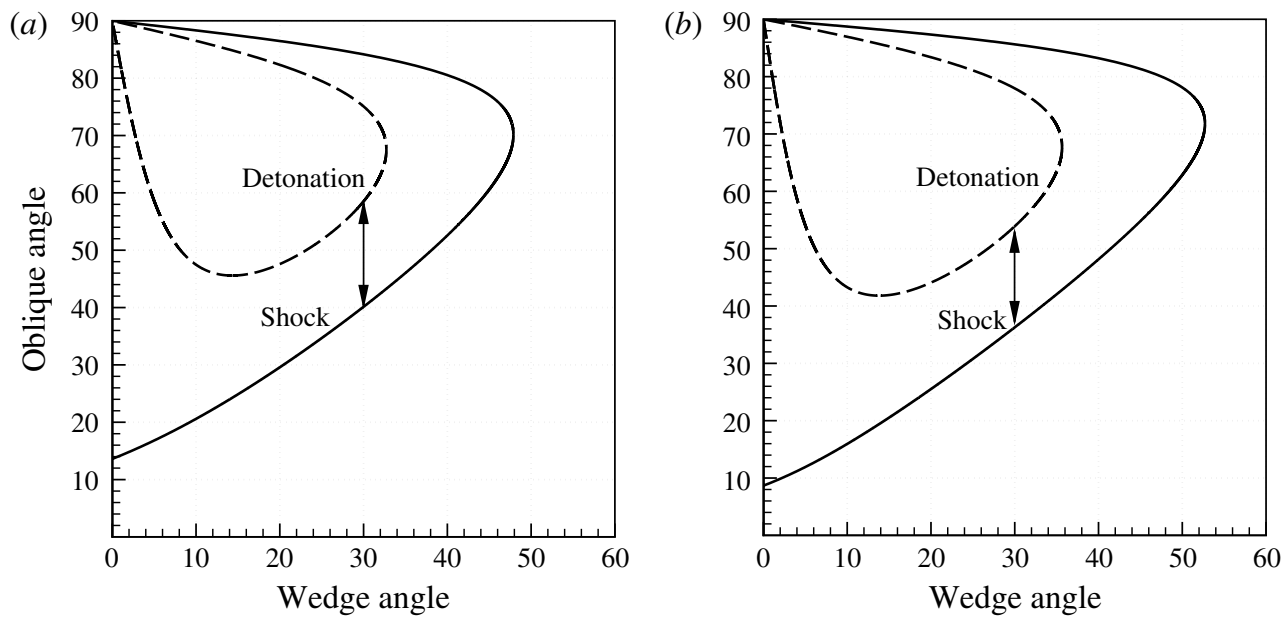

FIGURE 7. The shock and detonation polars with heat release $Q=8.33$ and inflow Mach ratio $(a) 1.4$ and $(b) 1.5$.

by the wedge angle $\theta$ and $M_{\text {in }}$ :

$$
\frac{\tan \beta_{1}}{\tan \left(\beta_{1}-\theta\right)}=\frac{(\gamma+1) M_{i n}^{2} \sin ^{2} \beta_{1}}{2+(\gamma-1) M_{i n}^{2} \sin ^{2} \beta_{1}} .
$$

The oblique detonation wave angle $\beta_{2}$ is

$$
\frac{\tan \beta_{2}}{\tan \left(\beta_{2}-\theta\right)}=\frac{(\gamma+1) M_{i n}^{2} \sin ^{2} \beta_{1}}{\gamma M_{i n}^{2} \sin ^{2} \beta_{2}+1-\sqrt{\left(M_{i n}^{2} \sin ^{2} \beta_{2}-1\right)^{2}-2\left(\gamma^{2}-1\right) M_{i n}^{2} \sin ^{2} \beta_{2} Q}} .
$$

Solving the above equations, the relation between the oblique angle and the wedge angle can be plotted as the shock/detonation polar. The shock and detonation polar curves in the case of $Q=8.33$ and $M_{i n} / M_{C J}=1.40$ and 1.50 are shown in figure 7 . The angle difference is large in the case of the abrupt transition as shown in figure $7(a)$, but small in the case of the smooth transition as shown in figure $7(b)$. For Mach ratio 1.40 the angles are $\beta_{1}=40.13^{\circ}$ and $\beta_{2}=58.51^{\circ}$, while for Mach ratio 1.50 the angles are $\beta_{1}=39.31^{\circ}$ and $\beta_{2}=53.90^{\circ}$. Although both $\beta_{1}$ and $\beta_{2}$ decrease when $M_{\text {in }}$ increases, the detonation deflection angle $\beta_{2}$ decreases quickly so the angle difference becomes small. Therefore it appears that the angle difference can be used as the criterion for the transition.

To check whether the angle difference is a universal criterion, results for the 12 cases are listed in table 2. It is found that the angle difference corresponding to $M_{c r}$ is located in a narrow region, between $14.90^{\circ}$ and $18.38^{\circ}$. This demonstrates that the angle difference is a proper criterion for the transition pattern. This is not surprising since it is determined physically by the flow structure. The gas mixtures across the oblique shock are ignited first on the wedge, and that will generate compression waves interacting with the oblique shock surface, as shown in figures 3-4. If the angle difference is small, the compression wave is enough to change the angle and subsequently induces the smooth transition. Otherwise, the abrupt transition is necessary. Comparing the results of cases 2 and 10-12, the angle differences are almost the same, which demonstrates the suitability of the criterion for different $Q$. 


$\begin{array}{lccc}\begin{array}{l}\text { Case } \\ \text { no. }\end{array} & M_{c r} & \beta_{2}-\beta_{1} \text { (deg.) } & t_{i} / t_{r} \\ 1 & & & \\ 2 & 1.44 & 16.61 & 0.424 \\ 3 & 1.46 & 15.73 & 0.443 \\ 4 & 1.48 & 15.20 & 0.462 \\ 5 & 1.45 & 16.20 & 0.436 \\ 6 & 1.47 & 15.53 & 0.452 \\ 7 & 1.49 & 14.90 & 0.466 \\ 8 & 1.42 & 17.44 & 0.430 \\ 9 & 1.41 & 17.89 & 0.427 \\ 10 & 1.40 & 18.83 & 0.423 \\ 11 & 1.47 & 15.87 & 0.464 \\ 12 & 1.49 & 15.94 & 0.488 \\ & 1.51 & 15.77 & 0.503\end{array}$

TABLE 2. Angle difference and time ratio for various $M_{c r}$ cases.

Thus, further evidence is provided to show that the angle difference is a reliable criterion.

Although the two kinds of transition patterns have been observed for a long time, research on the criterion has not attracted much attention. Papalexandris (2000) has studied the transition patterns and claimed that the smooth transition would appear in the case of large activation energy and small wedge angle. Wang, Zhao \& Jiang (2011) proposed that the transition pattern is determined by the post-shock flow Mach number. Their results are qualitatively the same as this research, but they do not provide a quantitative criterion. To the best of the authors' knowledge, the only operational criterion has been proposed by Figueira da Silva \& Deshaies (2000) from their numerical simulation using Navier-Stokes equations and the detailed chemical kinetic mechanism. Various cases are simulated with different wedge angle, temperature and pressure in their study. The ratio $t_{i} / t_{r}$ is proposed to be the criterion for the selection of the transition pattern, in which $t_{i}$ is the induction time and $/ t_{r}$ is the total reaction time. When $t_{i} / t_{r} \rightarrow 1$ the transition will be abrupt and when $t_{i} / t_{r} \rightarrow 0$ it will be smooth. The present numerical results are examined with this criterion as shown in table 2. It can be seen that the time ratio is about $0.4-0.5$ and the disparity is acceptable. However, $t_{i} / t_{r}$ does not give a good result for different heat release cases, comparing cases 2 and $9-10$. This is because the time ratio will change with the induction zone temperature (Figueira da Silva \& Deshaies 2000), and when the heat release varies the temperature also changes. In fact, the time ratio is a one-dimensional criterion, which is usually used in the detonation instability. Because the transition has different physical essence from the detonation instability, the time ratio is therefore not a suitable choice for the transition pattern criterion. The angle difference is a multi-dimensional, physical criterion, which should be a better choice than the time ratio.

\section{Conclusion}

Oblique detonation waves are simulated to study the OSW-ODW transition patterns with different gasdynamic and chemical parameters. There are two kinds of transition patterns observed: abrupt and smooth. The abrupt transition has a multi-wave point 
on the wave surface, while the smooth transition has a section of an arc shock. The abrupt transition has been studied widely, but the smooth transition and its relationship with the abrupt transition have not been fully addressed. In reality, both the structures may form and these may change into each other when the inflow parameters changes. Therefore a study on the operational criterion for the transition pattern is very important and helpful in ODWE design.

Qualitatively the abrupt transition will appear when $M_{\text {in }}$ is low and the smooth transition will appear when $M_{i n}$ is high. The critical Mach ratio $M_{c r}$, which corresponds to the upper limit of the abrupt transition, is defined to describe the transition pattern criterion. Numerical results show that $M_{c r}$ increases either when the temperature ratio $T_{B} / T_{S}$ increases, the activation energy $E_{B}$ decreases or the heat release $Q$ increases. Although the detonation instability is also influenced by these parameters, the transition pattern variation cannot be predicted according to the instability property. In this work, the angle difference is proposed as the criterion for determining the transition pattern with the aid of a polar analysis. The smooth transition will appear when the angle difference is small, while the abrupt transition will appear when the difference is large. The shift from the smooth to the abrupt transition occurs when the angle difference is about $15^{\circ}-18^{\circ}$. The results are also compared with previous results in the literature obtained with detailed chemical kinetic mechanism. The previously proposed time ratio criterion is examined and compared with the present angle difference criterion. It is found that the latter is proven to be better to discern the two types of transition patterns.

\section{Acknowledgements}

The authors are grateful for the help of $\mathrm{H}$. D. $\mathrm{Ng}$ of Concordia University. The research is supported by The National Natural Science Foundation of China NSFC No. 90916028.

\section{REFERENCES}

BRodA, J. C. 1993 An experimental study of oblique detonation waves. PhD thesis, University of Connecticut.

Choi, J. Y., Kim, D. W., Jeung, I. S., MA, F. \& YAnG, V. 2007 Cell-like structure of unstable oblique detonation wave from high-resolution numerical simulation. Proc. Combust. Inst. 31, 2473-2480.

Chol, J. Y., Shin, E. J. \& JeUnG, I. S. 2009 Unstable combustion induced by oblique shock waves at the non-attaching condition of the oblique detonation wave. Proc. Combust. Inst. 32, 2387-2396.

Daimon, Y. \& Matsuo, A. 2004 Analogy between wedge-induced steady oblique detonation and one-dimensional piston-supported unsteady detonation. Sci. Technol. Energ. Mater. 65, $111-115$.

Figueira Da Silva, L. \& Deshaies, B. 2000 Stabilization of an oblique detonation wave by a wedge: a parametric numerical study. Combust. Flame 121, 152-166.

Fusina, G., Sislian, J. P. \& Parent, B. 2005 Formation and stability of near Chapman-Jouguet standing oblique detonation waves. AIAA J. 43, 1591-1604.

Kailasanath, K. 2003 Recent developments in the research on pulse detonation engines. AIAA J. 41, 154-159.

Li, C., Kailasanath, K. \& Oran, E. S. 1993 Effects of boundary layers on oblique-detonation structures. AIAA Paper 1993-0450.

Li, C., Kailasanath, K. \& Oran, E. S. 1994 Detonation structures behind oblique shocks. Phys. Fluids 6, 1600-1611. 
Maeda, S., Inada, R., Kasahara, J. \& Matsuo, A. 2011 Visualization of the non-steady state oblique detonation wave phenomena around hypersonic spherical projectile. Proc. Combust. Inst. 33, 2343-2349.

Nettleton, M. A. 2000 The applications of unsteady, multi-dimensional studies of detonation waves to ram accelerators. Shock Waves 10, 9-22.

NG, H. D. \& LEE, J. H. S. 2003 Direct initiation of detonation with a multi-step reaction scheme. J. Fluid Mech. 476, 179-211.

PapaleXandris, M. V. 2000 Numerical study of wedge-induced detonations. Combust. Flame 120, 526-538.

Roy, G. D., Frolov, S. M., Borisov, A. A. \& Netzer, D. W. 2004 Pulse detonation propulsion: challenges, current status, and future perspective. Prog. Energy Combust. Sci. 30, $545-672$.

ShARPE, G. J. 2001 Transverse waves in numerical simulations of cellular detonations. J. Fluid Mech. 447, 31-51.

SHORT, M. \& QUIRK, J. J. 1997 On the nonlinear stability and detonability limit of a detonation wave for a model three-step chain-branching reaction. J. Fluid Mech. 339, 89-119.

Toro, E. F. 1999 Riemann Solvers and Numerical Methods for Fluid Dynamics. Springer.

Viguier, C., Figueira da Silva, L., Desbordes, D. \& Deshaies, B. 1997 Onset of oblique detonation waves: comparison between experimental and numerical results for hydrogen-air mixtures. Proc. Combust. Inst. 26, 3023-3031.

Vlasenko, V. V. \& SAbelnikov, V. A. 1995 Numerical simulation of inviscid flows with hydrogen combustion behind shock waves and in detonation waves. Combust. Explos. Shock Waves 31, 376-389.

WANG, A. F., ZHAO, W. \& JIANG, Z. L. 2011 The criterion of the existence or inexistence of transverse shock wave at wedge supported oblique detonation wave. Acta Mech. Sinica 27, 611-619. 